\title{
Ballistic Transport in Open Quantum Dots: Scar Wavefunctions and Resonance Line Shapes
}

\author{
M. Mendoza and P. A. Schulz \\ Instituto de Física Gleb Wataghin, UNICAMP, Cx.P. 6165, 13083-970, Campinas, SP, Brazil
}

Received on 4 April, 2005

\begin{abstract}
We present simulations of transport through highly transmitting open cavities within a lattice Green's function formalism. The qualitative relation between the line shape of the conductance and the symmetries of the corresponding scar wavefunctions is discussed. This system presents similar scar wavefunctions at different conductance plateaus. At the high plateau index limit the scars associated with bouncing ball classic orbits suffer a transition from accessible to quasi-unaccessible from outside the square billiard.
\end{abstract}

Keywords: Ballistic transport; Open quantum dots; Scar wavefunctions

Open quantum dots (OQD) with highly transmitting channels are systems where charging effects are not relevant and single-particle resonances should be robust. An example is experimentally described in the literature [1], for a regime of quantum dot sizes that are comparable to the Fermi wavelength $\lambda_{F}$, with a single entrance mode. OQDs structures with such characteristics are suitable for wavefunction imaging based on shifts in energy of the resonances, induced by AFM tip [2]. The same procedure could also be proposed for imaging of quantum billiard (QB) states or quantum dots that are much larger than $\lambda_{F}$ (high energy) and with several entrance modes $[3,4]$.

The transmission resonances are features of the quantum transport that can persist even in the semiclassical regime and are related to scar wavefunctions of regular periodic orbits, which are classically unaccessible from the leads. This suggests that dynamical phase-space tunneling may play an important role in the transport through open quantum cavities[5, 6]. For systems with mixed dynamics that comprise the majority of dynamical systems found in the nature a mechanism of chaos-assisted tunneling in multistep process has been suggested[7]. The assumptions that all dot energy levels are uniformly broadened by opening the leads and that only classical trajectories that connect the input and output leads contribute to conductance are not necessarily true for these systems: the electrons can stay in the dot for a rather long time in any periodic orbit associated with a resonance in the transmission[8] and in this case a fully quantum mechanical approach is necessary, a similar modeling is done by Akis and Ferry[4].

Our aim is to study the qualitative relation between the line shape of the conductance and the symmetries of the corresponding scar wavefunctions in a quantum-semiclassical limit for a open square billiard that presents a mixed dynamic at high energies.

The method used to simulate the structure consists in a hybridization between the self-energy technique [9] and the recursive method $[2,10]$. The total Halmitonian, $\left(H_{T}\right)$, can be writen as a function of the Hamiltonians, $H_{i i}$, of each transverse chain in which the system is divided, and using the selfenergies, $\Sigma^{L}$ and $\Sigma^{R}$, for describing the effects of the leads on the device (Fig. 1):

$$
H_{T}=\left(\begin{array}{cccccc}
H_{11}+\Sigma^{L} & V & 0 & 0 & . & . \\
V & H_{22} & V & 0 & . & . \\
0 & V & H_{33} & V & 0 & . \\
. & \cdot & \cdot & \cdot & \cdot & \cdot \\
. & \cdot & . & \cdot & \cdot & \cdot \\
. & . & . & . & . & H_{M M}+\Sigma^{R}
\end{array}\right)
$$

Here we are considering that the system is composed by $\mathrm{M}$ transverse chains with $\mathrm{N}$ sites each. Each transverse chain is described by:

$$
H_{i i}=\left(\begin{array}{cccccc}
\varepsilon_{1}^{i} & V_{y} & 0 & 0 & \cdot & . \\
V_{y} & \varepsilon_{2}^{i} & V_{y} & 0 & . & . \\
0 & V_{y} & \varepsilon_{3}^{i} & V_{y} & 0 & . \\
. & . & \cdot & \cdot & . & . \\
\cdot & . & . & . & . & \cdot
\end{array}\right)
$$

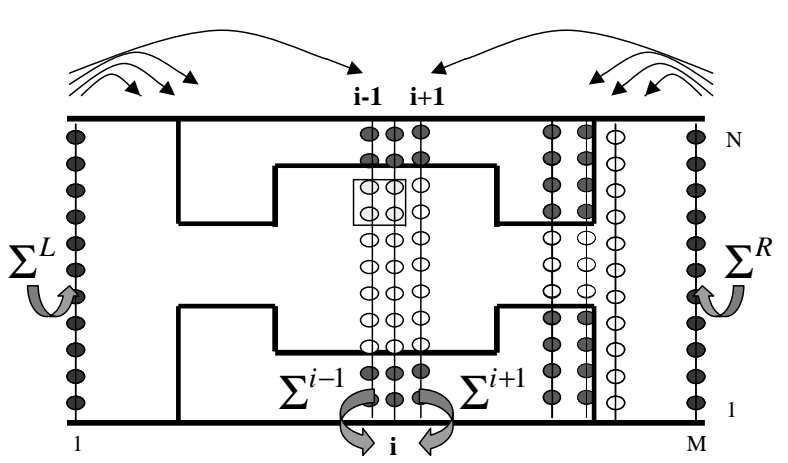

FIG. 1. Schematic illustration of the method and structure used.

The hopping sub-matrices in eq.(1), $V$, are diagonal, with elements $\langle i|V| i\rangle=V_{x}$.

In eq.(1) $\Sigma^{L}=V g_{L} V$ and $\Sigma^{R}=V g_{R} V$ ( $V$ is real) are the so called self-energies which describe the effects of the leads on the device, where $g_{L}$ and $g_{R}$ are the Green's functions for the isolated contacts:

$$
\left(E-H_{L}\right) g_{L}(E)=I \text { and }\left(E-H_{R}\right) g_{R}(E)=I
$$


Explicit expressions for $g_{L}$ and $g_{R}$ are shown elsewhere [2].

This formalism permits the use of conventional recursive Green's function method for calculating the conductance $[2,10]$. However, in this paper our main concern is the calculation of the local density of states (LDOS):

$$
\rho_{E}(m, n)=\frac{-1}{\pi} \operatorname{Im}\left[G_{T}(m, n ; E)\right]
$$

where $\mathrm{m}$ is the longitudinal site index, which define a transversal chain of $\mathrm{N}$ sites ( $\mathrm{n}$ is the transversal site index). In order to calculate this equation it is necessary to obtain the diagonal matrix elements of the total Green's function:

$$
G_{i i}=<i\left|\left[(E+i \eta) \mathbf{I}-H_{T}\right]^{-1}\right| i>
$$

which can not be calculated using the conventional recursive method. The necessary generalization of the method is the central issue of on discussion. In order to calculate $G_{i i}$, we start with the Hamiltonian of the corresponding transverse chain, $H_{i i}$ (in Fig. 1 some of these chains are illustrated) of $H_{T}$. The $i$-th chain divides the system in two sub systems, one at the left with $i-1$ chains and one to the rigth with $M-i$ chains. Next, we obtain the Green's functions of the sub systems projected on the $i-1$-th and $i+1$-th chains, by means of a recursive procedure:

$$
G_{m, m}=\left(1-G_{m, m}^{L} V G_{m+1, m+1}^{R} V\right)^{-1} G_{m, m}^{L}
$$

For the left sub system, $G^{L}$ is the iterated left chain, while $G^{R}$ is the added chain at each iteration. Similarly, the right sub system is handled by eq. (6) interchanging $L$ and $R$. This permits to calculate the self-energies:

$$
\Sigma^{a}=V^{+} G_{a} V
$$

at each side of $i(a=i-1$ and $a=i+1)$. These self-energies are added to $\left.H_{i i}\right)$ :

$$
G_{i i}=\left(E-H_{i i}-\Sigma^{i-1}-\Sigma^{i+1}\right)^{-1}
$$

This is the Green's functions of the $i$ transversal chain of the system or device, in other words, eq. (8) gives the diagonal matrix elements of the total Green's functions of the system. This method is specially useful for calculating the local density of states of large systems.

Close to realistic device dimensions are achieved, here we use up to $M=171$ (longitudinal sites) and $N=101$ (transversal sites) to define the structure represented schematically in Fig. 1. The parametrization of the electronic GaAs effective mass, $m^{*}=0,067 m_{0}$, leads to a value for the hopping parameters given by $V_{x, y}=-\hbar^{2} /\left(2 m^{*} a^{2}\right)=-0,142 \mathrm{eV}$ for a host lattice parameter of $a=20 \AA$. Hence, the OQD size is $L x=L y=101 a=0,2 \mu \mathrm{m}$. The QPCs are $L=25 a(500 \AA)$ long and $w=51 a(1000 \AA)$ wide (Fig. 1). These are already experimentally achievable dimensions.

For the purpose of illustrating the different effects in the transport, resonances in the conductance and classic orbits or scar wavefunctions, we have simplified the problem using a square OQD with hard wall confinement. The evolution of classic orbits and chaos with the rise of the incident electron energy shows a fingerprint in the conductance of the systems with an underlying regular classical dynamics. The dimensionless conductance curves vs incident energy $E$ shown in Fig. 2 are for regimes with (a) 5, (b) 10 and (c) 21 transmitting modes in the connecting quantum point contact (QPC) with a given width, simulating quantum dots that are much larger than $\lambda_{F}$ (high energy) with several entrance modes [3, 4].

Here, the conductance is characterized by narrow isolated resonances on top of a smooth background for $N$ transmitting modes, characteristic of OQDs at lower energies (Fig. 2(a)), similar to the $N=1$ case in the reference [2] or at high energies (Fig. 2(b-c)) when the underlying classical dynamics is mixed [11]. It is important here to observe the effect of the connecting OPCs:
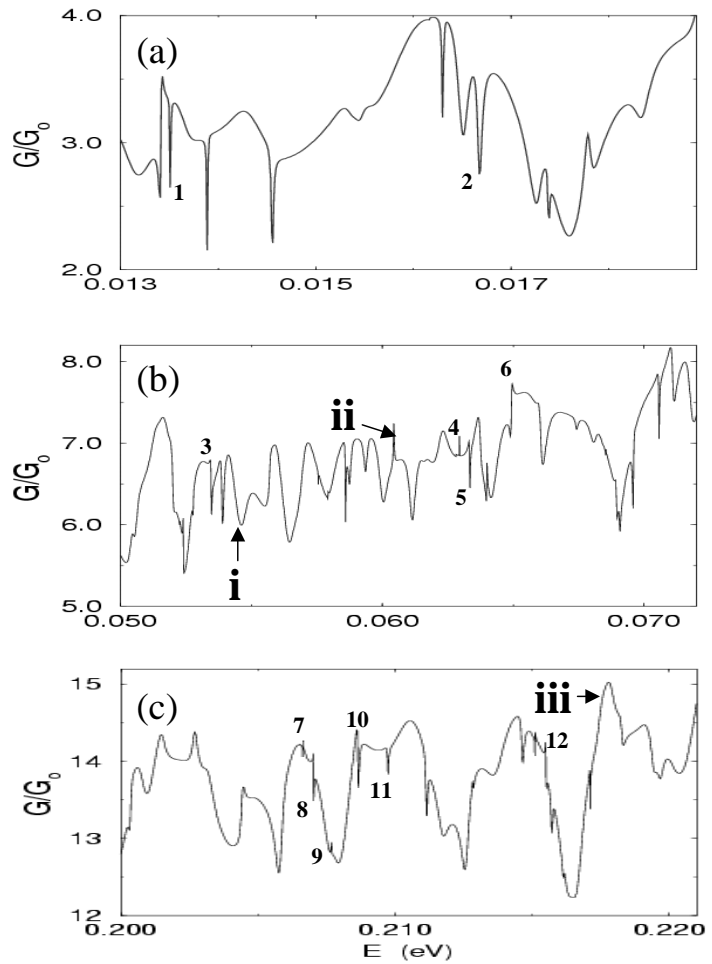

FIG. 2. Conductance as a function of electron incident energy of device shown in Fig. 1 for regimes with (a) 5, (b) 10 and (c) 21 transmitting modes for a fixed width of quantum point contacts.

the opening of the leads introduces and enhances chaos, in analogy to the opening of closed chaotic systems, where a rise in the number of regular orbits occurs [4]. The interference effects due to the square geometry and hard wall confinement generate the diminution of the smooth background associated to the conductance plateaus and this diminution rises with increasing energy, Fig. 2(a-c).

At the energy interval of fifth conductance plateau (Fig. 2(a)), a quantum regime with clear Fano resonances [12] is observed. The wide line widths indicate a strong and 
efective coupling to the leads continua. In the region of the tenth plateau (Fig. 2(b)) a strong rise in the number of resonances is observed, as expected, for a similar interval of energy. Now the fine line widths are associated to a diminution of the coupling to the leads continua. For high energies in the semiclassical limit (Fig. 2(c)), we have a rather unexpected diminution of the number of the resonances on the smooth background. This could be associated to a rise of chaos in the system because of the high number of acessible
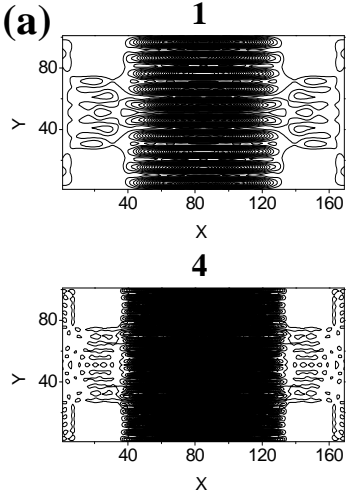

7
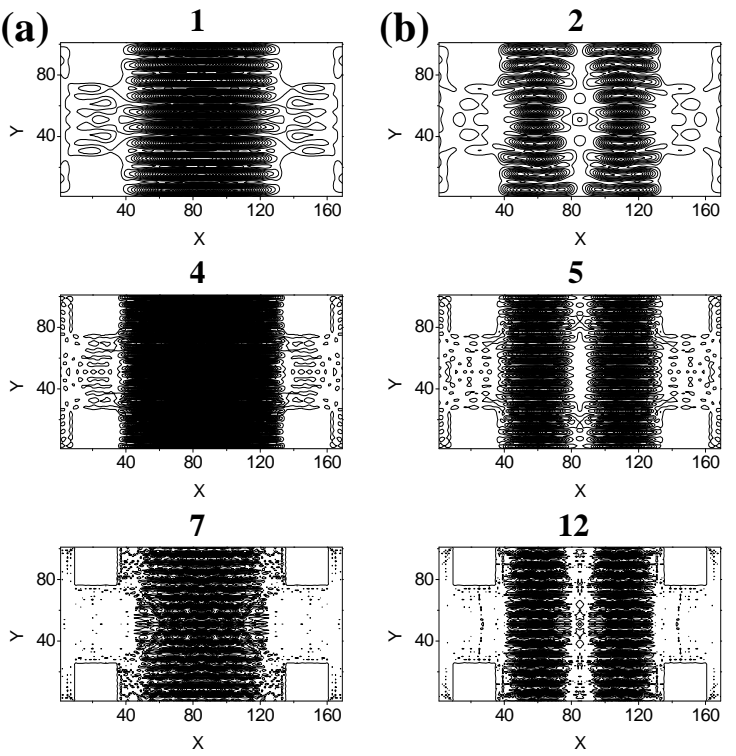

12

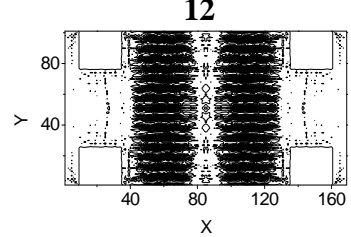

FIG. 3. Local density of states (LDOS) of the resonances 1,2,4,5,7,12 shown in the Fig. 2 associated with bouncing ball classic orbits. Here the evolution of the scars for classic orbits can be seen.

lead channels. The progressive suppression of the coupling to the continua is also observed. In order to verify these ideas we calculate the LDOS (Fig. 3) of the resonances observed in the Fig. 2. In Fig. 3 the effect of the diminution of $\lambda_{F}$ (rise of the energy) to simulate the evolution of classic orbits by means of scar wavefunctions in the quantum-semiclassical limit is shown. In this figure the LDOS of the resonances 1 and 2 observed in Fig. 2(a) are depicted. Here a clear bouncing ball-like scar wavefunction is observed with a strong coupling to continua. These scar wavefunctions present clear nodes, a fingerprint of quantum regime, for the two types of bouncing ball orbits (a) and (b), upper panels of the Fig. 3. The increase in energy increases the number of nodes of these scar wavefunctions (resonances 7 and 12, lower panels) leading to a better definition of the bouncing ball-like scar wavefunction. Specially in Fig. 3(b) we see that a typical lens shape modulation disappears, going from resonance 2 ( quantum limit) to resonance 12 (semiclassic limit). On the other hand, a diminution of coupling to the continua is observed by the suppression of the LDOS in the leads. These observations are evidences that the resonances associated to bouncing ball orbits, unaccessible from the leads at high energies, could become accessible by means of the chaotic states that show fingerprints in the conductance, Fig. 2(c)
[7]. This is a clear illustration of the quantum-semiclassical limit when the underlying classical dynamics is regular, but chaos

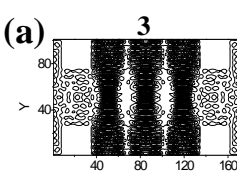

(b)

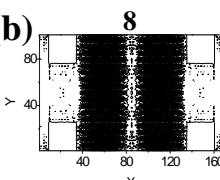

(c)

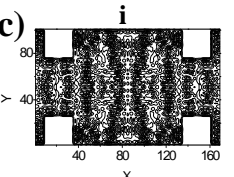

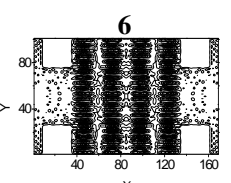
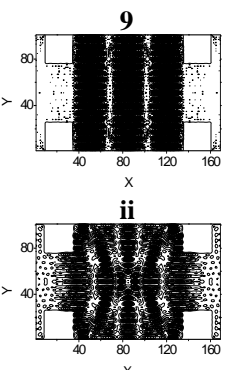

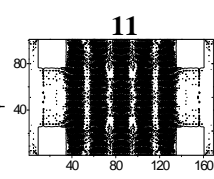

10

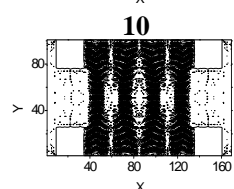

iii

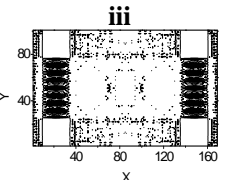

FIG. 4. Local density of states (LDOS) of the resonances (a) 3,6 and 11, (b) 8,9 and 10 shown in the Fig. 2 associated to bouncing ball classic orbits. (c) chaotic and continuum scar wavefunctions.

is generated by the opening of the system and plays an important role in the transport in absence of magnetic fields [4].

A sistematic analysis of the relation between the line shape of the conductance and the symmetries of the corresponding scar wavefunction can reveal which resonant states give a greater contribution to the transport. In the Fig. 2 all the resonances are asymmetric Fano-like indicating that the couplig to the discreete state in the dot and to the continua channels give similar contributions. Associated to these resonances we observe scar wavefunctions of classical bouncing ball-like with 1 and 2 anti-nodes (Fig. 3) and with 3,4 and 5 anti-nodes (Fig. 4(a-b)). All these resonances show a similar behaviour with increasing the number of transmitting modes. However, the evolution of the scars are different at diferents plateaus: states in the quantum limit may share the same energy range with others already in the semiclassic limit. For example, in Fig. 4(b), the resonance-10 (high energy) has a scar wavefunction with lens shaped nodes, characteristically from the quantum limit; while in Fig. 4(a), the resonance 6 (much lower energy) is associated to a scar wavefunction of the same kind, but already with characteristics of the semiclassic limit (well defined stripes without modulation). Finally in panel (c) of Fig. 4, other types of scars are observed. The i-resonance is a typical wide resonance with strong coupling to continuum of a bouncing ball-like orbit perturbed by the chaos. The resonant tunneling associated to the ii-resonance has a similar behavior associated to chaotic orbits. An example of a continuum state of the connecting QPCs is observed for the iii-resonance. Here it is clear that states of a closed system (quantum dot) with scar wavefunctions far from the entrances of open system can not couple to these continuum states and mechanisms associated to the presence of chaos can help in the coupling, via dynamical tunneling[7].

In summary, we have seen the evolution of scar wavefunctions in the quantum-semiclassical crossover, when the underlying classical dynamics is regular (for the corresponding 
closed system). In these systems the leads, or connecting QPCs, generate chaos at high energies that maintain the form of the resonance in the semiclassical limit. The generated chaos can be observed in the line shape of the conductance and the symmetries of scar wavefunctions. The Fano asymmetric resonances are bouncing ball classic orbits that suffer a transition of accessible to quasi-unaccessible, from outside the square billiard, with the rise of the energy.

This work has been supported by the Brazilian agencies FAPESP and CNPq. M.M. specially acknowledges financial support from FAPESP.
[1] T. Lundberg, J. E. F. Frost, K. F. Berggren, Z. L. Ji, C. T. Liang, I. M. Castleton, D. A. Ritchie and M. Pepper, Semicond. Sci. Technol.12, 875 (1997).

[2] M. Mendoza and P. A. Schulz, Phys. Rev. B 68, 205302 (2003).

[3] I.V. Zozoulenco et al., Phys. Rev. B 58, 10597 (1998).

[4] Jonathan P. Bird, Electron Transport in Quantum Dots (Kluwer Academic Publishers, 2003), p. 209.

[5] A. P. S. de Moura, Y. C. Lai, R. Akis, J. Bird, and D. K. Ferry, Phys. Rev. Lett. 88, 236804 (2002).

[6] A. Ramamoorthy, R. Akis, J. Bird, T. Maemoto, D. K. Ferry and M. Inoue, Phys. Rev. E 68, 026221 (2003).

[7] S. D. Frischat and E. Doron, Phys. Rev. E 57, 1421 (1998).

[8] Y. Takagaki and K.H. Ploog, Phys. Rev. E 62, 4804 (2000).

[9] S. Datta, Superlattices Microstruc. 28, 253 (2000).

[10] David K. Ferry and Stephen M. Goodnick, Transport in Nanostructures (Campbridge University Press, 1997), p. 156.

[11] B. Huckestein, R. Ketzmerick and C. H. Lewenkopf, Phys. Rev. Lett. 84, 5504 (2000).

[12] U. Fano, Phys. Rev. 124, 1866 (1961). 